\title{
Erratum to: Nanoparticles in the fight against mosquito-borne diseases: bioactivity of Bruguiera cylindrica-synthesized nanoparticles against dengue virus DEN-2 (in vitro) and its mosquito vector Aedes aegypti (Diptera: Culicidae)
}

\author{
Kadarkarai Murugan ${ }^{1}$ - Devakumar Dinesh ${ }^{1}$ - Manickam Paulpandi ${ }^{1}$. \\ Abdulaziz Dakhellah Meqbel Althbyani ${ }^{2}$. Jayapal Subramaniam ${ }^{1}$. \\ Pari Madhiyazhagan $^{1} \cdot$ Lan Wang $^{3}$ • Udaiyan Suresh ${ }^{1}$ • Palanisamy Mahesh Kumar ${ }^{1}$. \\ Jagathish Mohan ${ }^{1} \cdot$ Rajapandian Rajaganesh $^{1} \cdot \mathrm{Hui} \mathrm{Wei}^{4} \cdot \mathrm{Kandasamy} \mathrm{Kalimuthu}^{5}$. \\ Megha N. Parajulee ${ }^{6} \cdot$ Heinz Mehlhorn $^{7} \cdot$ Giovanni Benelli $^{8}$
}

Published online: 28 September 2015

(C) Springer-Verlag Berlin Heidelberg 2015

Erratum to: Parasitology Research DOI 10.1007/s00436-015-4676-8

In the original version of this article the captions of figures $2-5$ were inadvertently mixed-up. The corrected captions are as follows:

Figure 2. FTIR spectrum of silver nanoparticles biosynthesized using the Bruguiera cylindrica aqueous extract
Figure 3. SEM micrograph of silver nanoparticles biosynthesized using the Bruguiera cylindrica aqueous extract

Figure 4. EDX spectrum of silver nanoparticles biosynthesized using the Bruguiera cylindrica aqueous extract

Figure 5. XRD pattern of silver nanoparticles biosynthesized using the Bruguiera cylindrica aqueous extract

This correction does not alter the conclusion of the study.

The online version of the original article can be found at http://dx.doi.org/ 10.1007/s00436-015-4676-8.

Giovanni Benelli

g.benelli@sssup.it; benelli.giovanni@gmail.com

1 Department of Zoology, School of Life Sciences, Bharathiar University, Coimbatore 641046, Tamil Nadu, India

2 Faculty of Science, Department of Biology, University of Tabuk, Tabuk 71491, Saudi Arabia

School of Life Science and Technology, Shanxi University, Taiyuan 030006, China
4 Institute of Plant Protection, Fujian Academy of Agricultural Sciences, Fuzhou, Fujian 350013, China

5 Institute of Marine Biology, National Taiwan Ocean University, Keelung 20224, Taiwan

6 Texas A\&M University System/Agri Life Research and Extension Center, Lubbock, TX 79403, USA

7 Department of Parasitology, Heinrich Heine University, Düsseldorf, Germany

8 Department of Agriculture, Food and Environment, University of Pisa, via del Borghetto 80, 56124 Pisa, Italy 